\title{
Representation of competitions by generalized fuzzy graphs
}

\author{
Sovan Samanta ${ }^{1}$ Biswajit Sarkar $^{1}$ *, \\ ${ }^{1}$ Department of Industrial and Management Engineering, \\ Hanyang University, Ansan Gyeonggi-do, 15588, Korea. \\ E-mail: ssamantavu@gmail.com,bsbiswajitsarkar@gmail.com
}

Received 1 September 2017

Accepted 15 February 2018

\begin{abstract}
Generalized fuzzy graphs are perfect to represent any system like networks, images, scheduling, etc. compared to fuzzy graphs. This study introduces the concept of a generalized fuzzy neighbourhood of a vertex and generalized fuzzy graphs. Also, an associated graph, called minimal graphs of competition graphs are defined. These graphs will represent some practical competitions existing in the world. Some important results regarding the stated graphs are proved. Some area of applications in real life system is focused.
\end{abstract}

Keywords: Generalized fuzzy graphs, neighbourhood, competition, minimal graphs.

\section{Introduction}

Nowadays, graphs do not represent all the systems properly due to the uncertainty or haziness of the parameters of systems. For example, a social network may be represented as a graph where vertices represent an account (person, institution, etc.) and edges represent the relation between the accounts. If the relations among accounts are measured as good or bad according to the frequency of contacts among the accounts, fuzziness can be added for such representation. This and many other problems lead to define fuzzy graphs. The first definition of a fuzzy graph was by Kaufmann 14 in 1973 . But, it was Rosenfeld ${ }^{[28}$ who considered fuzzy relations on fuzzy sets and developed the theory of fuzzy graphs in 1975. Using this concept of a fuzzy graph, Koczy 16 used fuzzy graphs in the evaluation and optimiza- tion of networks. For further details of fuzzy graphs, readers may look in $9177|18| 19 \mid 27$.

There are several types of fuzzy graphs available in the literature. Intuitionistic fuzzy graph 20 , interval-valued fuzzy graphs 1, and bipolar fuzzy graphs ${ }^{2}$ are some of them. In all these fuzzy graphs, there is a common property that edge membership value is less than to the minimum of its end vertex membership values. Suppose, a social network is to be represented as fuzzy graphs. Here, all social units are taken as fuzzy nodes. The membership values of the vertices may depend on several parameters. Suppose, the membership values are measured according to the sources of knowledge. The relation between the units is represented by fuzzy edges. The membership value is measured according to the transfer of knowledge. But, transfer of knowledge may be greater than one of the social actors/units

\footnotetext{
* Corresponding author.

Email: bsbiswajitsarkar@gmail.com (Biswajit Sarkar).

Office Phone: +82-31-400-5259.

Mobile: +82-10-7498-1981. Fax: +82-31-436-8146.
} 
as more knowledgeable person informs less knowledgeable person. But, this concept cannot be represented in fuzzy graphs as edge membership value should be less than membership values of the end vertices. Thus, all images/networks cannot be represented by fuzzy graphs or any other types of fuzzy graphs. This study removes the restriction on edges.

In 1968, Cohen 11 introduced the notion of competition graphs in connection with a problem in ecology. Let $\vec{D}=(V, \vec{E})$ be a digraph, which corresponds to a food web. A vertex $x \in V(\vec{D})$ represents a species in the food web and an $\operatorname{arc} \overrightarrow{(x, s)} \in \vec{E}(\vec{D})$ means that $x$ preys on the species $s$. If two species $x$ and $y$ have a common prey $s$, they will compete for the prey $s$. Based on this analogy, Cohen defined a graph which represents the relations of competition among the species in the food web. The competition graph $C(\vec{D})$ of a digraph $\vec{D}=(V, \vec{E})$ is an undirected graph $G=(V, E)$ which has the same vertex set $V$ and has an edge between two distinct vertices $x, y \in V$ if there exists a vertex $s \in V$ and arcs $\overrightarrow{(x, s)}, \overrightarrow{(y, s)} \in \vec{E}(\vec{D})$.

The representation of competition by competition graphs does not show the characteristics properly. Samanta and $\mathrm{Pal} 23$ represented the competitions in a more realistic way. Also, they 22 showed that fuzzy graphs can be used in competition in ecosystems. The theory of competition graphs is further used in intuitionistic neutrosophic environment ${ }^{3}$, bipolar fuzzy graphs ${ }^{26}$, and others ${ }^{15}$, 4. Further, some works $5677877813 \mid 21$ on the topic increased the literature. But there was a restriction on edges. The membership value of edge which represents the strength of the relationship between preys and predator may be larger than its own membership values. More clearly, for example, a man whose strength is lower than a tiger can trap the tiger. This theory is developed by Sarkar and Samanta2624. Hence, generalized fuzzy graphs are more appropriate for the representation. In this study, generalized fuzzy competition graphs and related terms are defined.

After the introductory section, Problem definition is given in Section 2. After that, some basic notions are discussed in Section 3 . In that section, generalized fuzzy graphs of type 1 and type 2 (GFG1,
GFG2) are described with suitable examples. After that, in Section 4.1, the neighbourhood of vertices in GFG is defined. Generalized fuzzy competition graphs (GFCGs) are introduced in Section 4.2. A real-life application is represented through GFCG in Section 5. In Section 6, some benefits of this study are pointed. At last, a conclusion is drawn in Section 7.

\section{Problem definition}

Edge restriction of fuzzy graphs is generalized. The relation between membership values of vertices and edges are established. Also, this study focuses on a generalization of competition graphs. The competition graphs represent any kind of competitions in any system. But the aim of this study is to show the competition in the proper way including uncertainty and haziness. Besides, minimal graphs are introduced. These graphs are important to characterize the generalized competition graphs. At last, the graphs are applied to show the competitions among countries on the economy.

\section{Preliminaries}

A fuzzy set $A$ on a set $X$ is characterized by a mapping $m: X \rightarrow[0,1]$, which is called the membership function. A fuzzy set is denoted by $A=(X, m)$. The height of $A$ is $h(A)=\max \{m(x) \mid x \in X\}$.

A fuzzy graph $\xi=(V, \sigma, \mu)$ is a non-empty set $V$ together with a pair of functions $\sigma: V \rightarrow[0,1]$ and $\mu: V \times V \rightarrow[0,1]$ such that for all $x, y \in V$, $\mu(x, y) \leqslant \sigma(x) \wedge \sigma(y)$, where $\sigma(x)$ and $\mu(x, y)$ represent the membership values of the vertex $x$ and of the edge $(x, y)$ in $\xi$ respectively. A loop at a vertex $x$ in a fuzzy graph is represented by $\mu(x, x) \neq 0$. An edge is non-trivial if $\mu(x, y) \neq 0$.

The underlying crisp graph of $\xi=(\sigma, \mu)$ is denoted by $G^{*}=(V, E)$, where $V\left(G^{*}\right)=\{u \in V$ : $\sigma(u)>0\}$ and $E\left(G^{*}\right)=\{(u, v) \in V \times V: \mu(u, v)>$ $0\}$.

Now, generalized fuzzy graphs are defined. The membership values of vertices of graphs depend on membership values of the adjacent edges. The mem- 
bership values of isolated vertices are taken as 0 . The membership function is defined from a nonempty set to a closed interval $[0,1]$. Thus, any linguistic term can be defined by membership values. Sometimes, vertex membership values are considered first and depending on vertex membership values, the edge membership values are assumed. For example, social networks, where social actors and its stability are considered first. Depending on stability, vertex membership values are determined. After that, a relation among the actors is considered. The membership values may be taken from the parameter 'relationship'. Again, in some problems, edges are considered first and depending on edge membership values the vertex membership values are considered. For example, capacities of pipelines can be taken as edge membership values and depend on the capacities, vertex membership values are decided.

Here, two types of relations are considered. In the following, a generalized fuzzy graph of the first kind is defined. Here, vertex membership values are considered first. Then, depending on vertex membership values, edge membership values are considered.

Definition 1. Let $V$ be a non-empty set. Two functions are considered as follows. $\rho: V \rightarrow$ $[0,1]$ and $\omega: V \times V \rightarrow[0,1]$. Also, let $A=$ $\{(\rho(x), \rho(y)) \mid \omega(x, y)>0\}$. The triad $(V, \rho, \omega)$ is said to be generalized fuzzy graph of first kind (GFG1) if there exists a function $\phi: A \rightarrow(0,1]$ such that $\omega(x, y)=\phi((\rho(x), \rho(y)))$ where $x, y \in V$. Here $\rho(x), x \in V$ is the membership value of the vertex $x$ and $\omega(x, y), x, y \in V$ is the membership value of the edge $(x, y)$.

Now, generalized fuzzy graphs of second kind is defined. Here, the membership values of edges are considered first. Then, depending on edge membership values, vertex membership values of vertices are assigned.

Definition 2. ${ }^{24}$ Let $V$ be a non-empty set. Two functions are considered as follows. $\rho: V \rightarrow[0,1]$ and $\omega: V \times V \rightarrow[0,1]$. Also, let $B$ be the range set of $\omega$. The triad $(V, \rho, \omega)$ is said to be generalized fuzzy graph of second kind (GFG2) if there exists a function $\psi: B \rightarrow(0,1]$ such that for all $x \in V$, $\rho(x)=\psi\left(\omega\left(e_{x}\right)\right)$ where $e_{x}=(x, y)$ such that $y \in V$.
Here, $\rho(x), x \in V$ is the membership values of the vertex $x$ and $\omega(x, y)$ is the generalized membership value of the edge $(x, y)$.

Note 1 In GFG2, the co-domain set of $\psi$ excludes the number 0 , as the membership values of vertices are always positive.

\subsection{Generalized fuzzy directed graphs (GFDGs)}

The generalized directed fuzzy graph of first kind (GDFG1) is defined below.

Definition 3. Let $V$ be a non-empty set. Two functions are considered as follows. $\rho: V \rightarrow[0,1]$ and $\omega: \vec{E} \rightarrow[0,1]$, where $\vec{E}$ be a set of ordered elements of $V \times V . A=\{(\rho(x), \rho(y)) \mid \omega \overrightarrow{(x, y)}>0\}$. The triad $(V, \rho, \omega)$ is said to be generalized directed fuzzy graph of first kind (GDFG1) if there exists a function $\phi: A \rightarrow(0,1]$ such that $\omega \overrightarrow{(x, y)}=$ $\phi((\rho(x), \rho(y)))$ where $x, y \in V$. Here, $\rho(x), x \in V$ are membership value of the vertex $x$ and $\omega \overrightarrow{(x, y)}$ is the membership value of directed edge $\overrightarrow{(x, y)}$.

Example 1. Let the vertex set be $V=\{x, y, z, t\}$ and edge set be $\{\overrightarrow{(x, y)}, \overrightarrow{(x, z)}, \overrightarrow{(t, x)}, \overrightarrow{(y, t)}\}$. Also, let $\rho(x)=0.2, \rho(y)=0.9, \rho(z)=0.3, \rho(t)=0.8$. Let $\phi(m, n)=m \wedge n$. Here, $A=\{(0.2,0.9),(0.2,1.3)$, $(0.8,0.2),(0.9,0.8)\}$. Then $\omega \overrightarrow{(x, y)}=0.2 \wedge 0.9=$ $0.2, \omega \overrightarrow{(x, z)}=0.2, \omega \overrightarrow{(t, x)}=0.2, \omega \overrightarrow{(t, y)}=0.8$. The corresponding generalized fuzzy graph is shown in Fig 1

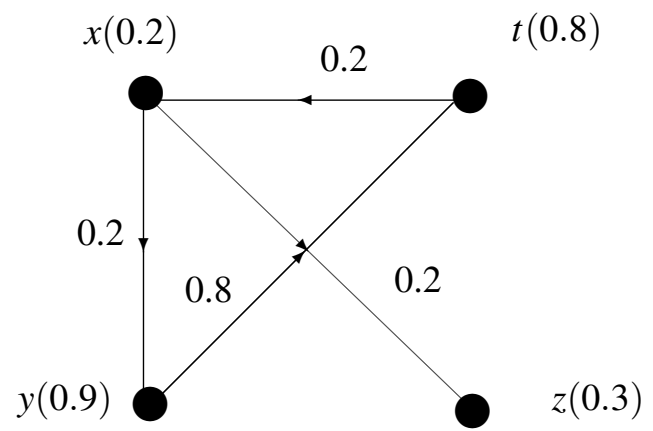

Fig. 1. A generalized directed fuzzy graph of first kind (GDFG1)

Degree of a vertex $x$ in GDFG1 is the pair $\left(d^{+}(x), d^{-}(x)\right)$ where $d^{+}(x)$, out degree of $x$, is the 
sum of the generalized membership values of outgoing edges and $d^{-}$, in degree of $x$, is the sum of the generalized membership values of incoming edges. Thus, $d^{+}(x)=\sum_{y \in V} \omega \overrightarrow{(x, y)}$ and $d^{-}(x)=$ $\sum_{y \in V} \omega \overrightarrow{(y, x)}$. In the Fig. 11, the degree of $x$ is $(0.2+0.2,0.2)=(0.4,0.2)$. The degree of the vertices $y, z, t$ are $(0.8,0.2),(0,0.2),(0.2,0.8)$ respectively.

Note 2 In GDFG1, out degree or in degree of a vertex may be 0 . But, when both are 0 then the vertex is called null vertex.

Generalized directed fuzzy graph of second kind (GDFG2) is introduced below.

Definition 4. Let $V$ be a non-empty set. Two functions are considered as follows. $\rho: V \rightarrow[0,1]$ and Let $\omega: \vec{E} \rightarrow[0,1]$, where $\vec{E}$ be a set of ordered elements of $V \times V$. Also, let $B$ be the range set of $\omega$. The triad $(V, \rho, \omega)$ is said to be generalized directed fuzzy graph of second kind (GDFG2) if there exists a function $\psi: B \rightarrow(0,1]$ such that for all $x \in V$, $\rho(x)=\psi\left(\omega\left(e_{x}\right)\right)$ where $e_{x}=\overrightarrow{(x, y)}$ or $e_{x}=\overrightarrow{(y, x)}$ such that $y \in V$.

From the definition it is clear that, if a vertex has no incoming edge but has outgoing edges, its generalized membership value is 0 . Let us discuss this situation with an example.

Example 2. Here, $V=\{a, b, c, d, e\}$. The edge membership values are given as $(\overrightarrow{(b, a)}, 0.5),(\overrightarrow{(d, a)}, 0.4),(\overrightarrow{(b, c)}, 0.6),(\overrightarrow{(c, d)}, 0.5)$, $(\overrightarrow{(e, c)}, 0.8),(\overrightarrow{(a, e)}, 0.7),(\overrightarrow{(d, e)}, 0.2)$. Here, $\psi(x)=$ $\max \{\omega \overrightarrow{(y, x)}\}, y \in V$. Now, the generalized membership value of the vertex $a$ is $\rho(a)=0.5+0.4=0.9$ as there are only two directed edges $\overrightarrow{(b, a)}, \overrightarrow{(d, a)}$. Similarly, other vertices have the weights as $b(0), c(1.4)$, $d(0.5), e(0.9)$. Thus, the graph of Fig. 2 is GDFG2.

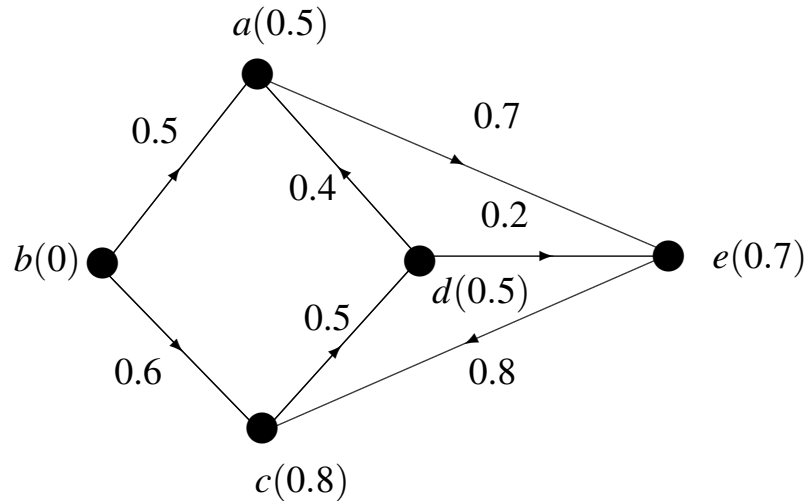

Fig. 2. A generalized directed fuzzy graph of second kind (GDFG2)

Degree of a vertex $x$ in GDFG2 (similarly defined) is the pair $\left(d^{+}(x), d^{-}(x)\right)$ where $d^{+}(x)$, out degree of $x$, is the sum of the generalized membership values of outgoing edges and $d^{-}$, in degree of $x$, is the sum of the generalized membership values of incoming edges. Thus, $d^{+}(x)=\sum_{y \in V} \omega \overrightarrow{(x, y)}$ and $d^{-}(x)=\sum_{y \in V} \omega \overrightarrow{(y, x)}$.

Note 3 In GDFG2, in-degree or out-degree of a vertex may be zero. If both of them are zero, the vertex is said to null vertex.

\subsection{Isomorphism in GFGs}

The definition of homomorphism is given first.

Definition 5. Let $\xi_{1}=\left(V_{1}, \rho_{1}, \omega_{1}\right)$ and $\xi_{2}=\left(V_{2}, \rho_{2}, \omega_{2}\right)$ be two GFG1 (or GFG2). Also let, $h: V_{1} \rightarrow V_{2}, \theta_{1}: A_{1} \rightarrow[0,1]$ and $\theta_{2}: A_{1} \rightarrow[0,1]$ be the functions where $A_{1}=$ $\left\{\rho_{2}(x) \mid x \in V_{2}\right\}$ and $A_{2}=\left\{\left(\rho_{2}(x), \rho_{2}(y)\right) \mid x, y \in\right.$ $V_{2}, \quad(\mathrm{x}, \mathrm{y})$ is an edge in $\left.\xi_{2}\right\}$ such that $\rho_{1}(x)=$ $\theta_{1}\left(\rho_{2}(h(x))\right)$ and $\omega_{1}(x, y)=\theta_{2}\left(\omega_{2}(h(x), h(y))\right)$. Then, the function $h$ is said to be homomorphism between two generalized fuzzy graphs.

If two systems are equal in vertex and edge membership values, then they are called isomorphic.

Definition 6. Let $\xi_{1}=\left(V_{1}, \rho_{1}, \omega_{1}\right)$ and $\xi_{2}=$ $\left(V_{2}, \rho_{2}, \omega_{2}\right)$ be two homomorphic GFG1s (or GFG2s). Also let, $h: V_{1} \rightarrow V_{2}$, be a bijective homomorphism and $\theta_{1}$ and $\theta_{2}$ are both identity mappings. Then, the function $h$ is said to be isomorphism between two generalized fuzzy graphs.

Example 3. Let us consider a GFG1, $\xi_{1}=$ 
$\left(V_{2}, \rho_{1}, \omega_{1}\right)$ where $V_{1}=\{a(0.6), b(0.5), c(0.7)$, $d(0.8)\}$ and edge set $\{((a, b), 0.5),((b, c), 0.5)$, $((c, d), 0.7), \quad((d, a), 0.8)\}$ which is shown in Fig. 3(a). Also, let another GFG1, $\xi_{2}=$ $\left(V_{2}, \rho_{2}, \omega_{2}\right) \quad V_{2}=\left\{a^{\prime}(0.6), \quad b^{\prime}(0.5), \quad c^{\prime}(0.7)\right.$, $\left.d^{\prime}(0.8)\right\}$ and edge set $\left\{\left(\left(a^{\prime}, b^{\prime}\right), 0.5\right),\left(\left(b^{\prime}, c^{\prime}\right), 0.5\right)\right.$, $\left.\left(\left(c^{\prime}, d^{\prime}\right), 0.7\right),\left(\left(d^{\prime}, a^{\prime}\right), 0.8\right)\right\}$. Also, let $h: V_{1} \rightarrow V_{2}$ such that $h(a)=a^{\prime}, h(b)=b^{\prime}, h(c)=c^{\prime}, h(d)=d^{\prime}$. $\theta_{1}(x)=x$ and $\theta_{2}(y)=y$. Then, by routine calculation, it is observed that the graphs are weak isomorphic.

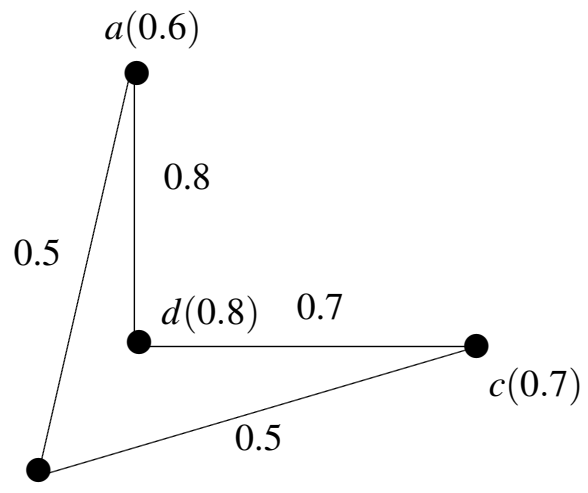

$b(0.5)$

(a): GFG1, $\xi_{1}$

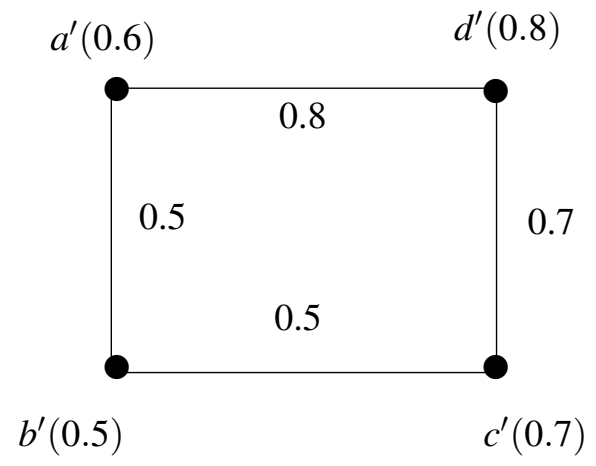

(b): A homomorphic GFG1, $\xi_{2}$

Fig. 3. Isomorphism between two generalized fuzzy graphs

\section{Generalized fuzzy competition graphs}

Neighbourhood of vertices is a related term to define GFCG.

\subsection{Neighbourhood of a vertex in GFG}

In GFG, neighbourhood of a vertex is the set of adjacent vertices. Let $x$ be a vertex of a GFG, $\xi$ and $x$ is adjacent to the vertices $y_{1}, y_{2}, \ldots, y_{k}$. Then, the neighbourhood of $x$ is the set $\left\{\left(y_{1}, \omega\left(x, y_{1}\right)\right),\left(y_{2}, \omega\left(x, y_{2}\right)\right), \ldots,\left(y_{k}, \omega\left(x, y_{k}\right)\right)\right\}$. A vertex $y$ is said to be strong neighbour of $x$ if $\omega(x, y)>0.5$. In GFDG, $\vec{\xi}$, a vertex $y$ is said to be out-neighbourhood of a vertex $x$ if there exists an edge $\overrightarrow{(x, y)}$ and the membership value of the out neighbourhood is $\omega \overrightarrow{(x, y)}$. Conversely, $x$ is said to be an in-neighbourhood of $y$ and the membership value of in-neighbourhood is $\omega \overrightarrow{(y, x)}$. A vertex $z$ is strong out/in-neighbourhood if $\omega \overrightarrow{(x, y)}>$ $0.5(\omega \overrightarrow{(y, x)}>0.5)$.

In Fig. 1, neighbourhood of $x$ is $\{(y, 0.9),(z, 0.5),(t, 0.8)\}$. In the same figure, the out-neighbourhood of $x$ is the set $\{(y, 0.2),(z, 0.2)\}$ and in-neighbourhood of $x$ is $(t, 0.2)$.

The neighbours may be indirect also. Suppose, there is a directed path from $x$ to $x_{m}$ of length $m$ as $x \rightarrow x_{1} \rightarrow x_{2} \rightarrow \ldots \rightarrow x_{m-1} \rightarrow x_{m}$. Then, $x_{m}$ is said to be $m$-step out neighbourhood of $x$. Let $N_{m}^{+}(x)$ be the set of $m$-step out neighbourhood of $x$. Thus, $x_{m} \in N_{m}^{+}(x)$. The membership value of $x_{m}$ in $N_{m}^{+}(x)$ is $\omega \overrightarrow{\left(x, x_{1}\right)} \times \omega \overrightarrow{\left(x_{1}, x_{2}\right)} \times \ldots \omega \overrightarrow{\left(x_{m-1}, x_{m}\right)}$. In Fig. 4 a directed graph is shown. Here $z_{2}$ is a 3-step out neighbourhood of $x$. So $z_{2}$ is an indirect neighbourhood of $x$. The membership value of $z_{2} \in N_{3}^{+}(x)$ is $0.9 \times 0.7 \times 0.8=0.504$. The concept of neighbourhood is generalized here. The direct or indirect (i.e. m-step) neighbourhoods are not mentioned in this case. If a vertex is both direct and indirect neighbourhood of another vertex, in generalized concept, indirect path may be taken, only if its membership value is greater than as direct neighbourhood. If a vertex $x$ is a $m_{1}, m_{2}, \ldots, m_{k}$ steps out neighbourhood of another vertex $y$, then the maximum membership value will be taken. The following example clarifies the issue. In Fig. 2, $e$ is the 1-step and 2-step out neighbourhood of $d$. Thus, $e$ with membership value 0.2 belongs to $N_{1}^{+}(d)$ and $e$ with membership value $0.4 \times 0.7=0.28$ belongs to $N_{2}^{+}(d)$. Thus, membership value of $e$ as a neighbourhood of $d$ is taken as 
0.28. This concept is used through out the paper, if no of steps are not mentioned.

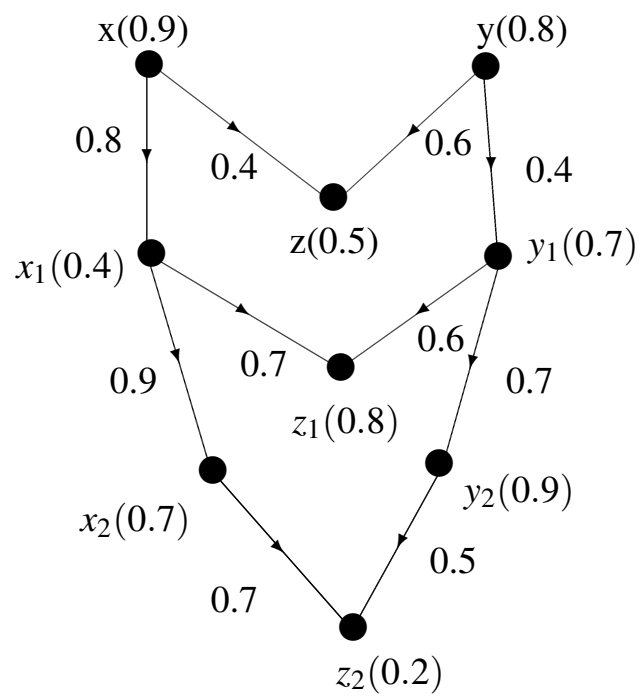

Fig. 4. Indirect neighbourhoods

\subsection{Generalized fuzzy competition graphs}

Definition 7. Let $\xi=(V, \rho, \omega)$ be a GFDG. Now, the generalized competition graph of $\xi$ is a GFG, $C(\xi)=\left(V, \rho^{\prime}, \omega^{\prime}\right)$ where $\rho^{\prime}=\rho$. Also, $\omega^{\prime}\left(x^{\prime}, y^{\prime}\right)=$ $h\left(N_{m}^{+}(x) \wedge N_{n}^{+}(y)\right)$ where $x^{\prime}, y^{\prime} \in C(\xi)$ are the corresponding vertices of $x, y \in \xi$ and $m, n$ are integers.

Example 4. Let us consider a competition among Tiger $(T), \operatorname{Lion}(L), \operatorname{Dog}(D)$, Bear $(B)$ for $\operatorname{Food}(F)$ (see Fig. 5). Here, the predators and prey are assumed as vertices, i.e. $V=\{T, L, D, B, F\}$. The membership values of the vertices are taken as their strengths for snatching the food. Suppose $\rho(T)=1, \rho(L)=0.9, \rho(D)=0.6, \rho(B)=$ $0.7, \rho(F)=0.35$. Also, the demands of the particular food for the predators are taken as edge membership values. Empirically, $\omega \overrightarrow{(T, F)}=0.95$, $\omega \overrightarrow{(L, F)}=0.85, \omega \overrightarrow{(D, F)}=0.45, \omega \overrightarrow{(B, F)}=0.35$ are taken. Thus, $h\left(N^{+}(T) \cap N^{+}(L)\right)=0.85$, $h\left(N^{+}(T) \cap N^{+}(D)\right)=0.45, h\left(N^{+}(T) \cap N^{+}(B)\right)=$ $0.35, \quad h\left(N^{+}(D) \cap N^{+}(L)\right)=0.45, \quad h\left(N^{+}(B) \cap\right.$ $\left.N^{+}(L)\right)=0.35, h\left(N^{+}(B) \cap N^{+}(D)\right)=0.35$. The corresponding GFCG is shown in Fig. 6

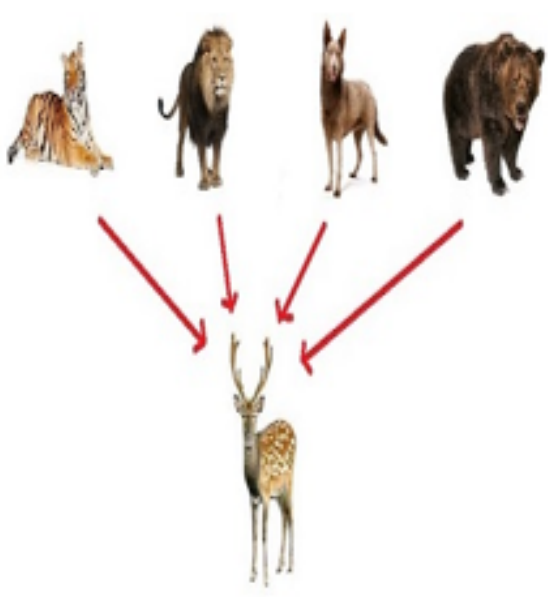

Fig. 5. Competition among predators. $L(0.9)$

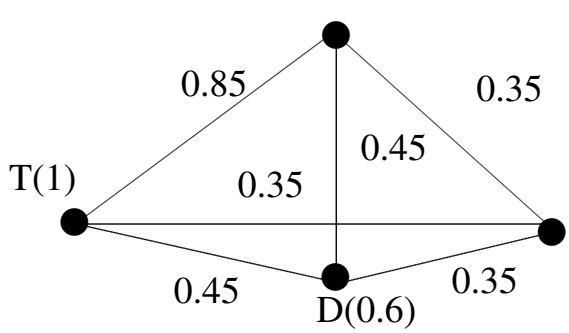

$\mathrm{B}(0.7)$

Fig. 6. GFCG of Fig. 5

GFCG represents any competition with much effective way. Any GFG may be considered as GFCG of some GFDG. This GFDG may not be unique. The following theorems clarify the statement.

Theorem 1. Let $\xi$ be a GFG. There exists a GFDG, $\vec{\varphi}$ such that $C(\vec{\varphi})=\xi$.

Proof. Let $\xi=(V, \rho, \omega)$ be a GFG and $(x, y)$ be an edge of the graph. Now, a GFDG, $\vec{\varphi}=\left(V, \rho^{\prime}, \omega^{\prime}\right)$ is to be constructed such that $C(\vec{\varphi})=\xi$. Let $x^{\prime}$ and $y^{\prime}$ be the corresponding vertices in $\vec{\varphi}$ of $x$ and $y$ with $\rho(x)=\rho^{\prime}\left(x^{\prime}\right), \rho(y)=\rho^{\prime}\left(y^{\prime}\right)$. Also, there is at least one common generalized out neighbourhood, say, $z^{\prime}$ of $x^{\prime}$ and $y^{\prime}$ such that $\omega(x, y)=h\left(N_{m}^{+}\left(x^{\prime}\right) \cap N_{n}^{+}\left(y^{\prime}\right)\right)$, $m, n$ are integers. This formation can be done empirically. Similarly, for all the vertices and edges of $\xi$, vertices can be formed as common out neighbourhoods in $\vec{\varphi}$. Thus, $C(\vec{\varphi})=\xi$. 
There may be more than one GFDGs which satisfies Theorem 1. An example is given below. In Fig. 7(a), a GFG is shown. In Fig. 7(b) and Fig. 7(c), GFDGs are shown whose GFCG is the same graph of Fig. 7(a). By routine calculation, this is easy to understand. So there are infinitely many GFDGs whose competition graph is $\xi$.

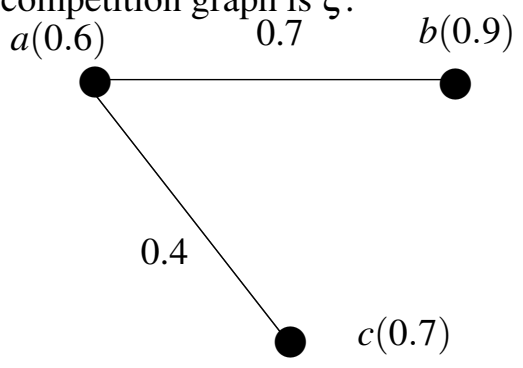

(a): A fuzzy graph, $\xi$

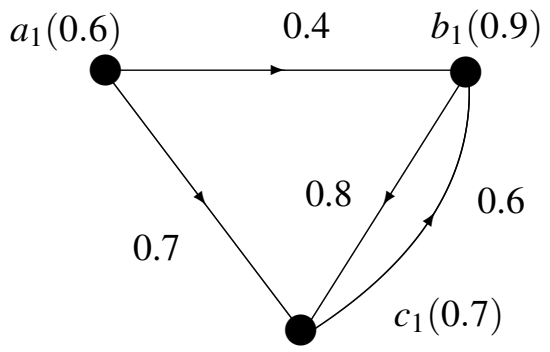

(b): A fuzzy directed graph, $\overrightarrow{\varphi_{1}}$

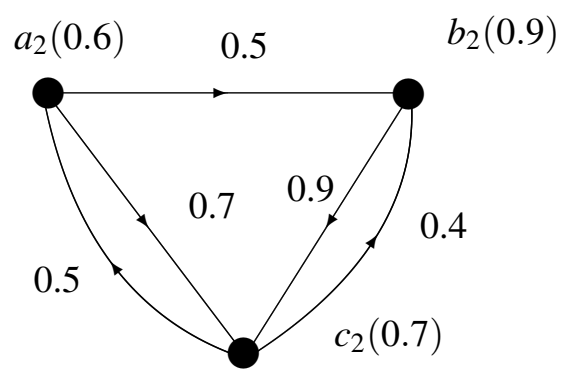

(c): A fuzzy directed graph, $\overrightarrow{\varphi_{2}}$

Fig. 7. GFDGs in (b), (c) and their GFCG in (a)

Among the GFDGs whose competition graph is a given GFG, some types of graphs have minimum number of edges. These graphs are called minimal graphs which are defined below.

Definition 8. Let $\xi$ be a GFG. Minimal graph, $\vec{\varphi}$ of $\xi$ is a GFDG, such that $C(\vec{\varphi})=\xi$ and $\vec{\varphi}$ has minimum number of edges, i.e. if there exists a GFDG $\overrightarrow{\varphi^{\prime}}$ other than $\vec{\varphi}$ such that $C\left(\overrightarrow{\varphi^{\prime}}\right)=\xi$, then number of edges in $\overrightarrow{\varphi^{\prime}}$ is greater than or equal to the number of edges in $\vec{\varphi}$.

Example 5. In Fig. 7(b), the number of edges of the graph, $\overrightarrow{\varphi_{1}}$ is 4 . And all other graphs, $\vec{\varphi}$ with the condition that $C(\vec{\varphi})=\xi$ has 4 or more edges. Thus, minimal GFDG of $\xi$ is $\vec{\varphi}$.

Minimal graphs of any GFG are not unique. Infinitely many GFDGs can be constructed with different edge membership values. But all the minimal graphs have same number of edges. The underline crisp graphs of the minimal graphs of a GFG are isomorphic. Thus, there exists one-one mappings between vertices and edges between the minimal graphs.

Theorem 2. Let $(x, y)$ be an edge in a $G F G$, $\xi=(V, \rho, \omega)$. Also, let $\overrightarrow{\varphi_{1}}=\left(V, \rho_{1}, \omega_{1}\right)$ be a minimal graph of $\xi$. Now, there exists a vertices $x_{1}, y_{1}, z_{1}$ in $\overrightarrow{\varphi_{1}}$ such that $\omega(x, y)=\omega_{1} \overrightarrow{\left(x_{1}, z_{1}\right)}$ or $\omega(x, y)=$ $\omega_{1} \overrightarrow{\left(y_{1}, z_{1}\right)}$.

Proof. Let $(x, y)$ be an edge in a GFG, $\xi=(V, \rho, \omega)$ and $\overrightarrow{\varphi_{1}}=\left(V, \rho_{1}, \omega_{1}\right)$ be a minimal graph of $\xi$. Minimal graphs are drawn in such a way that there exists common out neighbourhood, say $z_{1}$, of $x_{1}$ and $y_{1}$ where $x_{1}, y_{1}$ are the corresponding vertices of $x, y$ respectively. Thus, there are two directed edges $\overrightarrow{\left(x_{1}, z_{1}\right)}$ and $\overrightarrow{\left(y_{1}, z_{1}\right)} . \quad N^{+}\left(x_{1}\right)=\left\{\left(z_{1}, \overrightarrow{\omega_{1}} \overrightarrow{\left(x_{1}, z_{1}\right)}\right)\right\}$ and $N^{+}\left(y_{1}\right)=\left\{\left(z_{1}, \omega_{1} \overrightarrow{\left(y_{1}, z_{1}\right)}\right)\right\}$. Also, $\omega(x, y)=$ $h\left(N^{+}\left(x_{1}\right) \cap N^{+}\left(y_{1}\right)\right)$. Thus, $\omega(x, y)=\omega_{1} \overrightarrow{\left(x_{1}, z_{1}\right)}$ or $\omega(x, y)=\omega_{1} \overrightarrow{\left(y_{1}, z_{1}\right)}$.

Theorem 3. Isomorphic GFDGs are minimal graphs of some GFGs, but minimal graphs are not necessarily isomorphic.

Proof. The isomorphic graphs have equal number of edges. The membership values of corresponding vertices and edges of the isomorphic graphs are equal. Thus, if one GFDG is minimal graph of some GFGs, then all other isomorphic graphs are minimal. But, the converse may not be true. If $(x, y)$ is an edge in a GFG, $\xi=(V, \rho, \omega)$ and $\overrightarrow{\varphi_{1}}=\left(V, \rho_{1}, \omega_{1}\right)$ is a minimal graph of $\xi$. Now, there exists vertices $x_{1}, y_{1}, z_{1}$ in $\overrightarrow{\varphi_{1}}$ which are corresponding to $x, y, z$ in $\xi$ such that $\omega(x, y)=\omega_{1} \overrightarrow{\left(x_{1}, z_{1}\right)}$ or 
$\omega(x, y)=\omega_{1} \overrightarrow{\left(y_{1}, z_{1}\right)}$ i.e $\overrightarrow{\left(x_{1}, z_{1}\right)}$ or $\overrightarrow{\left(y_{1}, z_{1}\right)}$ have the same membership value as $(x, y)$. Without loss of generality, let us assume that $\omega(x, y)=\omega_{1} \overrightarrow{\left(x_{1}, z_{1}\right)}$. Also, let $\overrightarrow{\varphi_{2}}=\left(V, \rho_{2}, \omega_{2}\right)$ be another minimal graph of $\xi$ and $x_{2}, y_{2}, z_{2}$ are the vertices such that the edge $\overrightarrow{\left(x_{2}, z_{2}\right)}$ of $\overrightarrow{\varphi_{2}}$ is corresponding to $\overrightarrow{\left(x_{1}, z_{1}\right)}$ of $\overrightarrow{\varphi_{1}}$. Now, $\quad \overrightarrow{\omega_{1}} \overrightarrow{\left(x_{1}, z_{1}\right)} \wedge \omega_{1} \overrightarrow{\left(y_{1}, z_{1}\right)}=\omega_{2} \overrightarrow{\left(x_{2}, z_{2}\right)} \wedge$ $\omega_{2} \overrightarrow{\left(y_{2}, z_{2}\right)}$. This does not imply that $\omega_{1} \overrightarrow{\left(x_{1}, z_{1}\right)}=$ $\omega_{2}\left(x_{2}, z_{2}\right)$. Hence, minimal graphs are not necessarily isomorphic.

The characterization of minimal graphs such as the number of possible edges are discussed below.

Theorem 4. Let $\xi$ be a given connected GFG whose underlying graph is a cycle with $m$ edges. The number of edges in minimal graphs of $\xi$ is equal to $2 \mathrm{~m}$ where $m \geqslant 3$.

Proof. Let $\xi$ be a given connected GFG whose underlying graph is a cycle with $m$ edges. Let $x_{1}, x_{2}, \ldots, x_{m}$ be the vertices of $\xi$. Also, let $y_{1}, y_{2}, \ldots, y_{m}$ be the corresponding vertices in a minimal graph $\vec{\varphi}$. For every edge $(x, y)$ in $\xi$, there will be only one common out neighbourhood of $y_{1}$ and $y_{2}$ in $\vec{\varphi}$, say $y_{3}$. Also, $y_{3}$ is not common out neighbourhood of any other vertices in $\vec{\varphi}$. Similarly, $y_{2}, y_{3}$ has only one common out neighbourhood, say $y_{4}$ and so on (see Fig. 8). Thus for $m$ edges in $\xi$, there exists $2 m$ edges in $\vec{\varphi}$.

Theorem 5. Let $\xi$ be a given connected GFG whose underlying graph is a complete graph with $n$ vertices. The number of edges in minimal graphs of $\xi$ is equal to $2 n$ where $n \geqslant 3$.

Proof. Let $\xi$ be a given connected GFG whose underlying graph is a complete graph of $n$ vertices. Every vertices of $\xi$ are connected with each others. Let $x, y$ be two adjacent vertices in $\xi$ and $x_{1}$ and $y_{1}$ be the corresponding vertices in the minimal graph $\vec{\varphi}$. Now, if a GFDG, say $\overrightarrow{\varphi_{1}}$, is considered in such a way that every vertices of $\vec{\varphi}$ other than $x_{1}$ has only out neighbourhood as $x_{1}$ (see Fig. 9). Then, the GFDG has $n-1$ edges. Similarly, if $y_{1}$ is considered in place of $x_{1}$ in another GFDG, $\overrightarrow{\varphi_{2}}$, the number of edge will be same i.e. $n-1$. Now, consider a directed graph $\overrightarrow{\varphi_{3}}$ which has only two edges namely $\overrightarrow{\left(x_{1}, z_{1}\right)}$ and $\overrightarrow{\left(y_{1}, z_{1}\right)}$. In general, membership values of vertices and edges are taken as per needs of the proof. Now, consider the graph $\overrightarrow{\varphi_{1}} \cup \overrightarrow{\varphi_{2}} \cup \overrightarrow{\varphi_{3}}$. It is easy to observe that $\vec{\varphi}=\overrightarrow{\varphi_{1}} \cup \overrightarrow{\varphi_{2}} \cup \overrightarrow{\varphi_{3}}$. Thus the number of edges in $\vec{\varphi}$ is $(n-1)+(n-1)+2=2 n$.

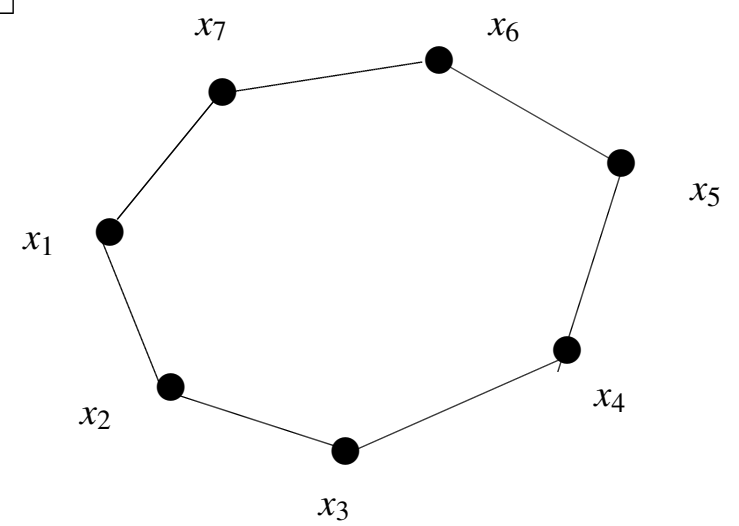

(a): A generalized fuzzy graph $\xi$.

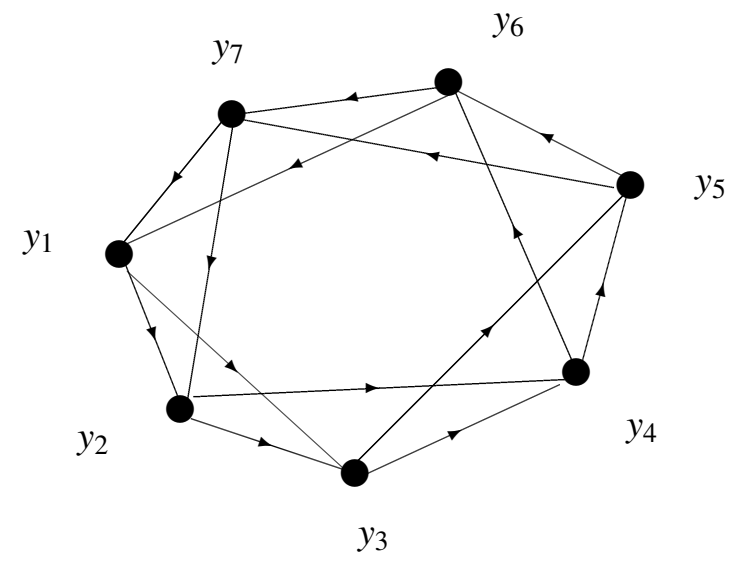

(b): A minimal graph of $\xi$

Fig. 8. A GFG with $m$ edges and its minimal graph with $2 m$ edges

It is an important task to calculate the number of edges in a minimal graph of a given graph. It is easy to prove that the number of edges of a minimal graph is less than or equal to $2 m$ if the given graph has $m$ edges.

Note 4 Let $\xi$ be a given connected GFG with $m$ edges. The number of edges in minimal graphs of $\xi$ is less than or equal to $2 m$ where $m \geqslant 4$.

Let $\xi$ be a connected GFG with $m$ edges where $m \geqslant 4$. If underline graph of $\xi$ is a cycle, then the number of edges in minimal graph is twice the number of edge in $\xi$. Again, underline graph of $\xi$ is 
complete graph, then number of edge is twice the number of vertices. For $n \geqslant 4$, the number of edges of such GFG is greater than its number of vertices. This concludes the result.

Note 5 Let $\xi$ be a given connected GFG with $m$ edges where $m \leqslant 3$. The number of edges in minimal graphs of $\xi$ is equal to $2 m$.
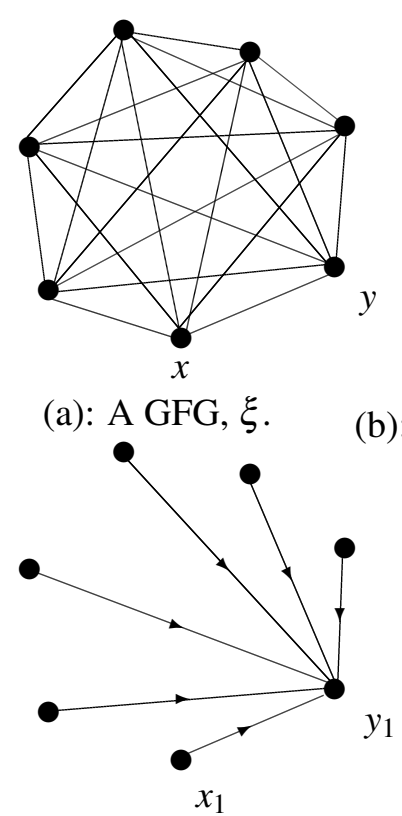

(c): A fuzzy subgraph, $\overrightarrow{\varphi_{2}}$

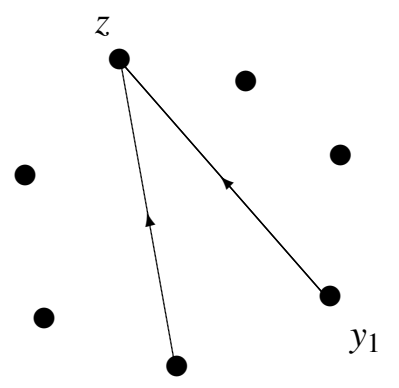

(d): A fuzzy subgraph, $\overrightarrow{\varphi_{3}}$

Fig. 9. A GFG (whose underling graph is complete) with $n$ vertices and its minimal graph with $2 n$ edges.

\section{Real life problem by GFCG}

Competition is arising in every aspect of life. Here an example of real life competition among the countries for economic growth is given. The countries $\{$ France(F), India(I), USA(U), Russia(R), China(C),
Germany $(\mathrm{G})\}$ are considered here. In Fig. 10, the competition is shown. The graphical representation of the competition is shown in Fig. 11. (a). Now, the competition among the countries is shown in Fig. 11 (b). Suppose the competition between $U$ and $C$ is to be calculated. In Fig. 11 (a), $U$ has direct edge to $\mathrm{E}$ (economy) with membership value 0.8 and has indirect edge (2-step) $0.8 \times 0.5=0.4$. So out neighbourhood of $U$ is $E$ with membership value 0.8 . Now, $C$ has direct edge to $E$ with membership value 0.65 and indirect edge with membership value $0.9 \times 0.8=0.72$. Thus out neighbourhood of $C$ is $E$ with membership value 0.72 . As indirect out neighbourhood of $C$ is strong, so its membership is considered. Hence, there is a competition between $U$ and $C$. The membership value of the edge between $U$ and $C$ in corresponding competition graph is 0.72 according to the Definition 7. And all other edges in corresponding competition graph is shown in Fig. 11(b).

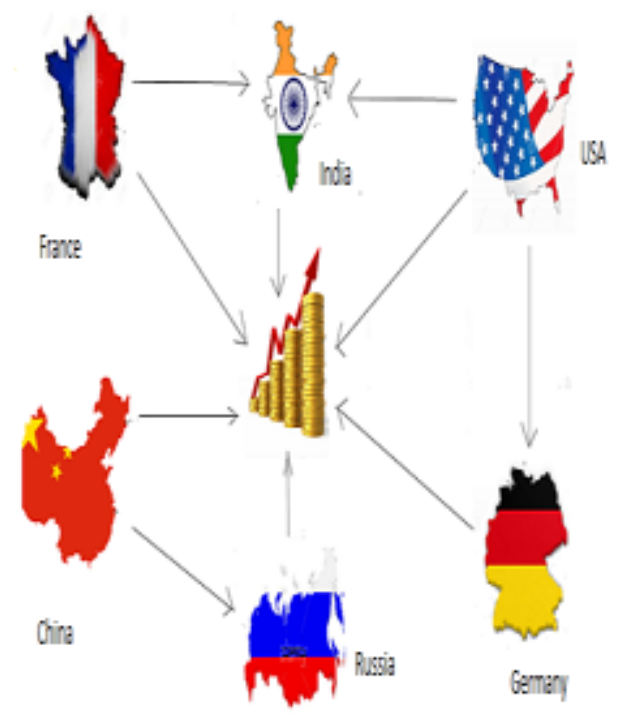

Fig. 10. Competition for economy among some countries

\section{Insights for this study}

- Edge restrictions on fuzzy graphs are removed

- The relations between vertex and edge membership values are established.

- The concept of neighbourhood is modified and more generalized. 
- Competition graphs are generalized.

- Minimal graphs are introduced. Some competitions are almost same. These competitions are represented by minimal graphs.

- An example is shown in Example 4 to show preypredators competition in forest.

- Some theorems are proved regarding minimal graphs. These theorems are helpful to build algorithms to find the number of vertex and edges in a minimal graphs of any large competitions.

- An example is shown in Section 5 to show the competition on economy among different countries.

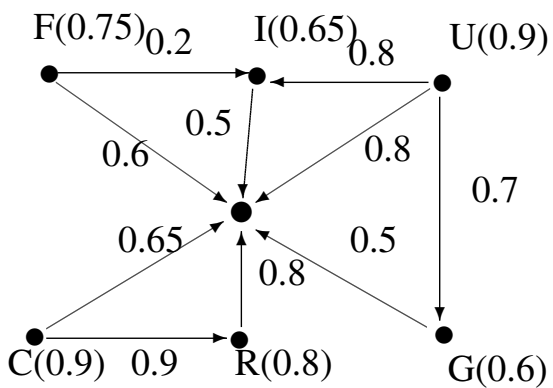

(a): Generalized fuzzy directed graphs

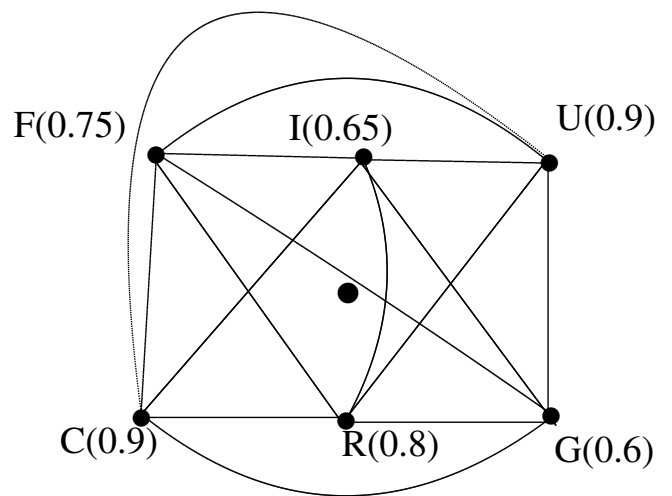

(b): Generalized fuzzy competition graphs

Fig. 11. Generalized fuzzy competition graphs

\section{Conclusions}

This study described some major properties of generalized fuzzy directed graphs. The concepts of neighbourhood of vertices were generalized for those graphs. Indirect neighbourhood may get preference over direct neighbourhood if the product of membership values of edges in indirect path is greater than membership value of direct edge. After that, concept of competition graphs was generalized. This study also introduced another important graph namely minimal graphs and their properties. The concept of minimal graph depended on number of edges. But, the concept can be extended depending on membership values of edges also. These representations are helpful to understand the real life competitions. In near future, the important properties and related algorithms will be established. Also, multi-label competitions must be represented by some graphs. This paper will be backbone for that.

1. M.Akram and W. A. Dudek, Interval valued fuzzy graphs, Computers and Mathematics with Applications, 61,289-299, 2011.

2. M. Akram, Bipolar fuzzy graphs, Information Sciences, doi:10.1016/j.ins. 2011.07.037, 2011.

3. M. Akram and M. Nasir, Certain competition graphs based on intuitionistic neutrosophic environment, Information 2017,8, 132; doi:10.3390/info8040132, 2017.

4. M. Akram and M. Sarwar, Novel applications of mpolar fuzzy competition graphs in decision support system,Neural Computing and Applications, DOI: 10.1007/s00521-017-2894-y, 2017

5. G. A. Azim, S. A. Khalek, A. S. F. Obada, A novel edge detection algorithm for image based on nonparametric fisher information measure, Applied and Computational Mathematics, 14(3), 316-327, 2015.

6. R. C. Brigham and R. D. Dutton, On neighbourhood graphs, Journal of Combinatories, Information, and System Sciences, 12, 75-85, 1987.

7. R.C. Brigham, F. R. McMorris and R. P. Vitray, Tolerance competition graphs, Linear Algebra and its Application, 217, 41-52, 1995.

8. C. Cable, K. F. Jones, J. R. Lundgren and S. Seager, Niche graphs, Discrete Apllied Mathematics, 23(3), 231-241, 1989.

9. M. T. Chien, S. T. Fang and Y. X. Su, A Generalization of Gershgorin Circles, Applied and Computational Mathematics, 15(1), 101-111, 2016.

10. H. H. Cho, S. R. Kim and Y. Nam, The $m$-step competition graph of a diagraph, Discrete Applied Mathematics, 105(1-3), 115-127, 2000.

11. J. E. Cohen, Interval graphs and food webs: a finding and a problem, Document 17696-PR, RAND Corporation, Santa Monica, CA (1968). 
12. M.C. Cooper, The tractability of segmentation and scene analysis, International Journal of Computer $\mathrm{Vi}$ sion, 30(1), 27-42, 1998.

13. R. D. Dutton and R. C. Brigham, A characterization of competition graphs, Discrete Applied Mathematics 6, 315-317, 1983.

14. A. Kauffman, Introduction a la Theorie des Sousemsembles Flous, Paris: Masson et Cie Editeurs, 1973.

15. M. G. Karunambigai, M. Akram, S. Sivasankar, and K. Palanivel, Clustering algorithm for intuitionistic fuzzy graphs, International Journal of Uncertainty, Fuzziness and Knowledge-Based Systems, 25(3), 367383, 2017

16. L. T. Koczy, Fuzzy graphs in the evaluation and optimization of networks, Fuzzy Sets and Systems, 46, 307-319, 1992.

17. S. Mathew and M.S. Sunitha, Types of arcs in a fuzzy graph, Information Sciences, 179, 1760-1768, 2009.

18. S. Mathew and M.S. Sunitha, Strongest strong cycles and theta fuzzy graphs, IEEE Transections on Fuzzy Systems, 2013.

19. J. N. Mordeson and P. S. Nair, Fuzzy Graphs and Hypergraphs, Physica Verlag, 2000.

20. R. Parvathi, M. G. Karunambigai, Intuitionistic Fuzzy Graphs, Computational Intelligence, Theory and Applications, 38, 139-150, 2006.
21. T Pramanik, S. Samanta, B. Sarkar and M. Pal, Fuzzy $\phi$-tolerance competition graphs, Soft Computing, 10.1007/s00500-015-2026-5, 2016

22. S. Samanta, M. Pal and M. Akram, $m$-step fuzzy competition graphs, Journal of Applied Mathematics and Computing, DOI 10.1007/s12190-014-0785-2, 2014.

23. S. Samanta and M. Pal, Fuzzy $k$-competition graphs and $p$-competition fuzzy graphs, Fuzzy Engineering and Information, 5(2), 191-204, 2013.

24. S. Samanta, B. Sarkar, D. Shin and M. Pal, Completeness and regularity of generalized fuzzy graphs, Springerplus, 5 (1979), 2016.

25. B. Sarkar and S. Samanta, Generalized fuzzy trees, International Journal of Computational Intelligence Systems, 10, 711-720, 2017.

26. M. Sarwar and M. Akram, Certain algorithms for computing strength of competition in bipolar fuzzy graphs, International Journal of Uncertainty, Fuzziness and Knowledge- Based Systems, 25(6), 877-896, 2017.

27. S. N. Shahbazova, Application of fuzzy sets for control of student knowledge, Applied and Computational Mathematics, 10(1), 195-208, 2011.

28. A. Rosenfeld, Fuzzy graphs, in: L.A. Zadeh, K.S. Fu, M. Shimura (Eds.), Fuzzy Sets and Their Applications, Academic Press, New York, 77-95, 1975. 